\title{
ИНДИКАТИВНО-ТЕМПОРАЛЬНОЕ ЛИНГВИСТИЧЕСКОЕ ОПИСАНИЕ МНОГОСЦЕНАРНЫХ РЕГИОНАЛЬНЫХ СОЦИАЛЬНО-ЭКОНОМИЧЕСКИХ \\ ПРОГРАММ
}

\section{INDICATIVE-TEMPORAL LINGUISTIC DESCRIPTION OF MULTI-SCENARIO REGIONAL SOCIO-ECONOMIC PROGRAMS}

\section{A. Lomazov}

Summary. Within the framework of the problematic of digital intellectual support of regional and municipal administration, the problem of constructing formal information models of programs for the socio-economic development of regions is considered. Taking into account the high level of uncertainty inherent in socioeconomic processes and requiring the involvement of experts for their assessment, it was proposed to use the apparatus of linguistic modeling by L. Zade to describe the programs. A complex of linguistic variables has been developed, reflecting the temporal and indicative aspects of the programs. Decision rules for the selection of sub-scenarios based on the system of fuzzy productions are proposed. The developed formal descriptions can be used in the development of management decision support systems when creating and administering the implementation of regional socio-economic programs.

Keywords: regional socio-economic program, scenario, linguistic variable, fuzzy production rule, fuzzy inference, decision support.

\author{
Ломазов Александр Вадимович \\ Аспирант, ФГАОУ ВО «Белгородский \\ государственный национальный исследовательский \\ университет» \\ lomazov_a@bsu.edu.ru
}

Аннотация. В рамках проблематики цифровой интеллектуальной поддержки регионального и муниципального администрирования рассмотрена задача построения формальных информационных моделей многосценарных программ социально-экономического развития регионов. С учетом высокого уровня неопределенности, присущего социально-экономическим процессам и требующего привлечения экспертов при их оценивании, для описания программ предложено использовать аппарат лингвистического моделирования Л. Заде. Разработан комплекс лингвистических переменных, отражающих темпоральные и индикативные аспекты программ. Предложены основанные на системе нечетких продукций решающие правила по выбору подсценариев. Разработанные формальные описания могут быть использованы при разработке систем поддержки принятия управленческих решений при создании и администрировании выполнением региональных социально-экономических программ.

Ключевые слова: региональная социально-экономическая программа, сценарий, лингвистическая переменная, нечеткое продукционное правило, нечеткий логический вывод, поддержка принятия решений.

личественный анализ сводится в основном к построению корреляционных и регрессионных соотношений между показателями социально-экономического развития регионов [9]. Между тем актуальной является задача развития современного интеллектуального инструментария разработки и анализа хода реализации РСЭП, позволяющего учесть высокий уровень не только статистической, но и лингвистически-нечеткой неопределенности, характерной для социально-экономической сферы.

Целью настоящей работы является развитие формального индикативно-темпорального аппарата для лингвистического модельного описания многосценарных РСЭП с использованием методов теории лингвистических переменных, нечеткого логического вывода на основе продукционной модели знаний о предметной области и теории принятия решений. 


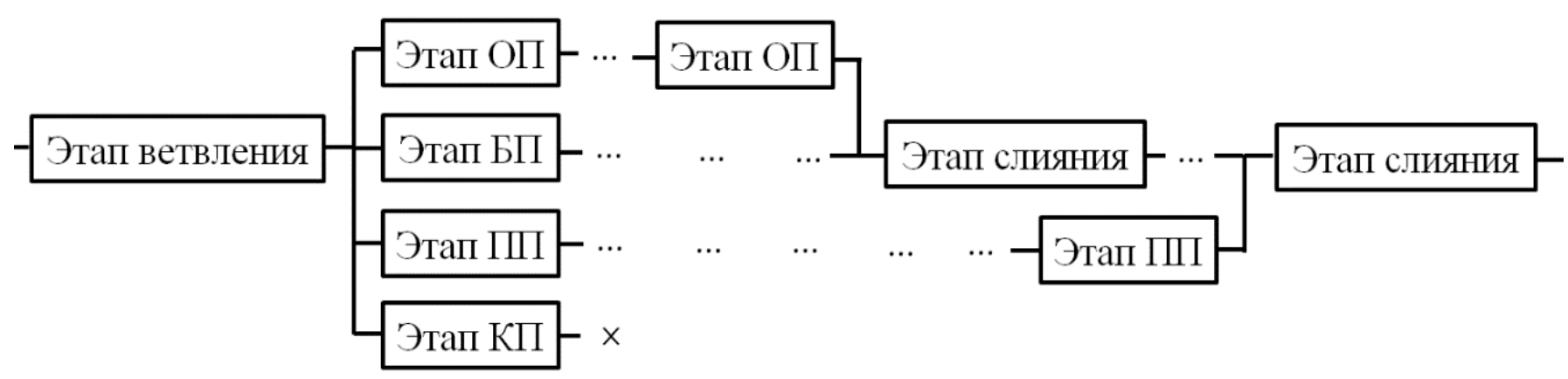

Рис. 1. Графическое представление фрагмента структурной модели РСЭП с учетом ограничений Огр. 1 - Огр.5

\section{Структурная темпора^ьная момель квазимногосшенарной РСЭП}

В качестве темпоральной модели будем рассматривать описание, ограниченное изучением только временных характеристик процесса (явления) [10] заданию времени начала и окончания каждого этапа. Темпоральная структурная модель односценарной РСЭП имеет линейное графическое представление в виде последовательности этапов. Многосценарность предполагает наличие этапов ветвления, после которых возможна реализация нескольких различных подсценариев, что приводит к графическому представлению многосценарной РСЭП в виде дерева сценариев (понимаемого в математическом плане, как связанный ориентированный граф без петель и контуров, вершинами которого являются этапы РСЭП: корень - начальный этап, листья - завершающие этапы, сценарии - различные пути от корня до листьев). С учетом специфики РСЭП сформулируем упрощающие предположения, ограничивающие рассматриваемый класс графических структурных моделей:

- Огр.1: эквиинициальность и эквифинальность (все сценарии имеют общий начальный и общий завершающий этап);

- Огр.2: наличие базового сценария (при проектировании РСЭП выделен основной сценарий, на реализацию которого рассчитаны имеющиеся ресурсы; все остальные сценарии рассматриваются как отклонения от базового сценария);

- Огр.3: ограниченность ветвлений (этапы ветвления могут находиться в составе только базового сценария);

- Огр.4: ограниченность слияний (подсценарии, начинающиеся с этапа ветвления завершаются одним из этапов базового сценария, расположенным до очередного этапа ветвления);

- Огр.5: ограниченность подсценариев (каждый этап ветвления является началом четырех ти- пов подсценариев: оптимистического, базового, пессимистического и катастрофического).

Графическое представление фрагмента структурной модели РСЭП с учетом ограничений Огр.1 - Oгр.5 и выделением в рамках ветвления оптимистического подсценария (ОП), базового подсценария (БП), пессимистического подсценария (ПП) и критического подсценария (КП), при котором выполняется один завершающий этап без достижения цели РСЭП представлено на рисунке 1. Необходимо отметить, что (как и показано на рисунке) слияние ОП с БП и слияние ПП с БП может происходить на разных этапах базового сценария.

Суть ограничений состоит в рассмотрении многовариантности сценариев как следствия отклонений от базового сценария, которые могут быть выявлены на некоторых этапах (этапах ветвления), после чего производится корректировка, состоящая в изменении нескольких последующих этапов с последующим возвращением к базовому сценарию (исключение составляет катастрофический подсценарий). Следует отметить, что корректировка целесообразна не только в случае неполного достижения цели этапа ветвления (пессимистический подсценарий), но и при превышении целевых показателей (оптимистический подсценарий), поскольку нарушение планового баланса показателей может создать риски, несоизмеримые с внеплановым выигрышем. Многосценарный подход к описанию РСЭП, при котором выполняются ограничения Огр.1 - Огр.5, назовем квазимногосценарным подходом. Центральным вопросом при построении квазимногосценарной РСЭП является задание решающих правил по выбору подсценариев, начинающихся с этапа ветвления. Переход на тот или иной подсценарий реализации РСЭП естественно производить в зависимости от показателей, достигнутых на этапе ветвления, что приводит к необходимости расширения темпорального описания РСЭП до индикативно-темпорального модельного описания. 


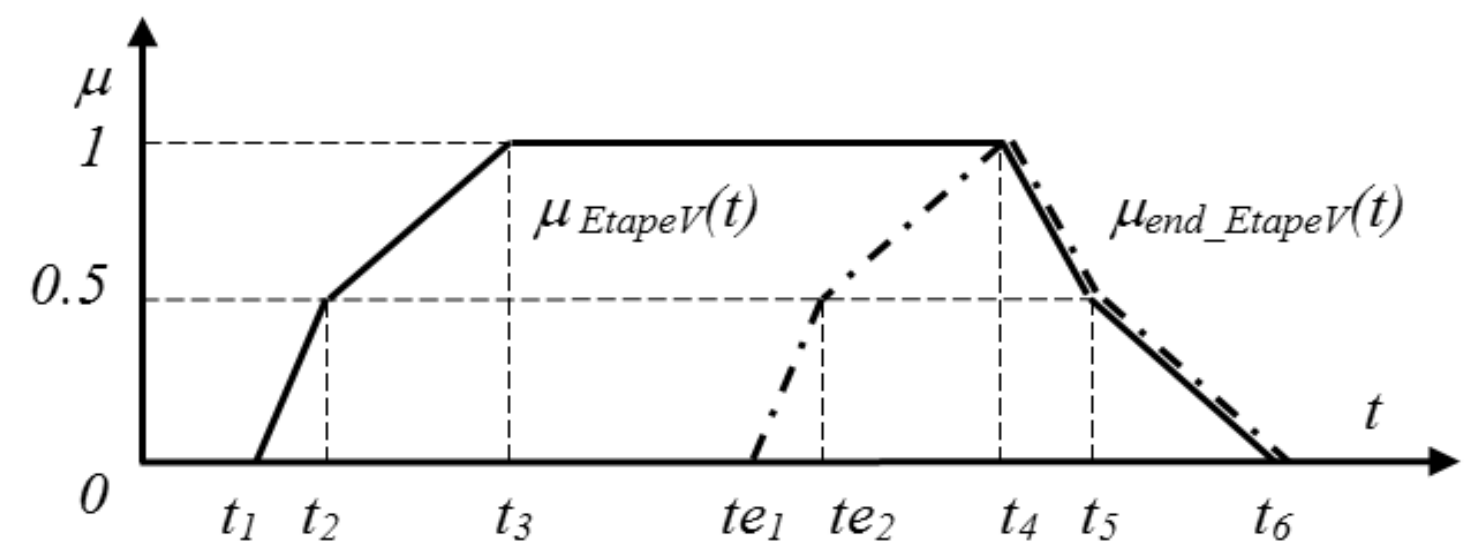

Рис. 2. Функции принадлежности термов EtapeV и end_EtapeV лингвистической переменной Etape

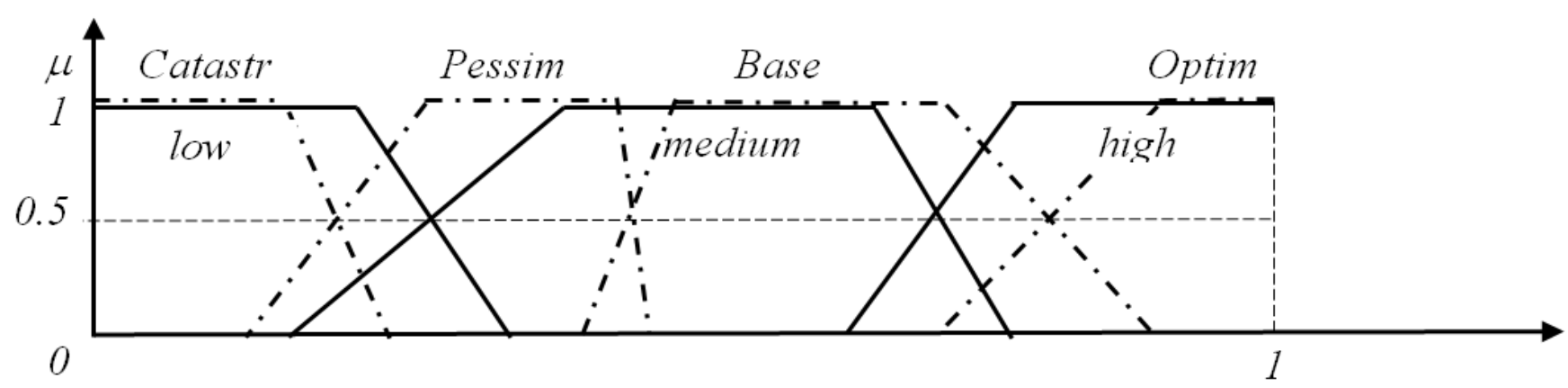

Рис. 3. Функции принадлежности термов low, medium, high лингвистической переменной Indi (сплошная линия) и термов Optim, Base, Pessim, Catastr лингвистической переменной Contr_Ind (штрих-пунктирная линия)

\section{ИнАикативно-темпоральное описание этапов РСЭП}

Будем рассматривать этап базового сценария EtapeV, на котором происходит ветвление, как один из базовых термов лингвистической переменной формально задаваемой (в соответствии с [11]) кортежем

$$
<\text { Stage, } T, T_{\text {Base }}, G, M>\text {, }
$$

где Stage - наименование лингвистической переменной «Этап РСЭП»; $T$ - временной континуум; $T_{\text {Base }}$ - базовый вектор темпоральных термов; $G-$ синтаксическое правило, позволяющее порождать наименования темпоральных термов из наименований элементов $T_{\text {Basei }} M$ - семантическое правило устанавливающее соответствие между темпоральными термами и нечеткими подмножествами $T$.

Например, синтаксическое правило < end_«базовый терм»>, примененное к терму EtapeV, имеющему семантику, определяемую функцией принадлежности $\mu \operatorname{EtapeV}(t)$ (показана на рисунке 2 сплошной линией) порождает терм < end_EtapeV $>$ (окончание этапа ветвления), имеющий семантику, определяемую функцией принадлежности $\mu$ end_EtapeV $(t)$ (показана на рисунке 2 штрих-пунктиром).

На рисунке $2 t 1, t 2, \ldots, t 6$ - задаваемые экспертами параметры функции принадлежности $\mu$ Etape $V(t)$ (имеющей битрапециевидный тип, предложенный для описания семантики темпоральных термов в [10]). Соответствующие параметры функции принадлежности $\mu$ end_EtapeV $(t): t e 1$, te 2, te $3=t e 4=t 4$, te $5=t 4$, te $6=e 6$.

Наряду с темпоральным описанием этапа EtapeV, рассмотрим набор относительных числовых индикативных социально-экономических показателей indl, ind $2, \ldots$, indn, принимающих значения в диапазоне от 0 до 1 и измеряемых в моменты времени, относящиеся к этапу EtapeV. Каждому из рассматриваемых показателей indi поставим в соответствие лингвистическую переменную Indi с единым для всех индикативных показателей базовым терм-множеством \{low, 
medium, high\}, но с разным заданием семантики базовых термов, отражающих лингвистически задаваемый уровень значений этих показателей. Функции принадлежности базовых термов low, medium, high индикативного показателя имеют традиционный вид, графически представленный на рисунке 3. На этом же рисунке пунктиром представлены также трапециевидные функции принадлежности термов Optim, Base, Pessim и Catastr лингвистической переменной Contr_Ind интегрального контрольного показателя, определяющего по какой из ветвей (подсценариев ОП, БП, ПП или КП) будет развиваться реализация РСЭП. Универсальным множеством переменной также является отрезок $[0,1]$.

Отметим, что индикативные показатели измеряются в момент времени, соответствующий окончанию этапа ветвления, тогда как значение интегрального контрольного показателя определяется (выводится) на основе измеренных значений индикативных показателей в рамках процедуры поддержки принятия решений по выбору подсценария реализации РСЭП.

Поддержка принятия решений по выбору подсценария РСЭП на основе индикативно-темпорального описания этапа ветвления

Пусть в результате измерений (и/или по данным статистической отчетности) имеются отражающие выполнение РСЭП на этапе ветвления значения индикативных показателей ind $1 *$, ind $2 *, \ldots$, ind ${ }^{*}$ в моменты времени $t 1^{*}, t 2 *, \ldots, t n *$, соответствующие окончанию этого этапа.

Решающее правило по выбору подсценария РСЭП запишем в виде системы нечетких продукций

(nOptim) если FOptim, mо (Contr_Ind-Optim)

(n Base) если FBase, mo (Contr_Ind-Base)

(nPessim) если FPessim, mo (Cöntr_Ind-Pessim)

(nCatastr) если FCatastr, mo (Contr_Ind-Catastr)

Здесь пOptim, пBase, пPessim, пCatastr - степени достоверности соответствующих продукционных правил, принимающие значения от 0 до 1 и вычисляемые с учетом исходной достоверности правил и соответствия времени измерения индикативных показателей лингвистическому терму. Например, при равном 1 значении степени исходной достоверности правила (полная достоверность) степень достоверности первого правила системы вычисляется по формуле:

$\eta$ Optim $=\left(\mu e n d \_E t a p e V(t 1 *)\right) \wedge\left(\mu e n d \_E t a p e V(t 2 *)\right) \wedge$ $\ldots \wedge\left(\mu\right.$ end_EtapeV $\left.\left(t^{*}\right)\right)$ где конъюнкция может пониматься в качестве одной из Т-норм (например, как min-норма).

Входящие в антецеденты правил нечеткие логические функции FOptim, FBase, FPessim, FCatastr имеют в качестве аргументов нечеткие высказывания относительно соответствия значений ind 1 , ind $2 *, \ldots$, indn* лингвистическим термам low, medium, high. Например, low).

FOptim $=\left(\right.$ ind $1 *_{-}$high $) \wedge\left(\right.$ ind $2 *_{-}$high $) \wedge\left(\right.$ ind $3 *_{-}$

Конкретный вид функций определяется в каждом конкретном случае особенностями РСЭП, и они могут рассматриваться (вместе с построенным набором лингвистических переменных) в качестве модели представления знаний о предметной области РСЭП.

Для выбора подсценария на основе системы продукционных правил может быть использовано два подхода. Первый подход предполагает использование алгоритма нечеткого логического вывода Мамдани [13], на последнем шаге которого (шаге дефаззификации) определяется числовое значение интегрального контрольного показателя contr ind. В зависимости от попадания этого значения в тот или иной подинтервал отрезка $[0,1]$ выбирается соответствующий подсценарий.

Второй подход состоит в использовании алгоритма Мамдани без выполнения шага дефаззификации. В этом случае лицу принимающему решение (ЛПР) предоставляется информация относительно каждого возможного подсценария с указанием степени соответствия результатов этапа ветвления этому подсценарию, а также степени достоверности используемого при этом правила. В случае высокой ответственности принимаемых управленческих решений с учетом присущего РСЭП высокого уровня неопределенности второй подход представляется более предпочтительным, поскольку он не только предлагает ЛПР рекомендуемый вариант, но дает также дополнительную информацию о степени его обоснованности, оставляя окончательное решение ЛПР с учетом опыта, интуиции и неформализуемых предпочтений.

\section{Зак^ючение}

В настоящей работе в рамках развития формального индикативно-темпорального аппарата для лингвистического модельного описания многосценарных РСЭП:

- предложена структурная темпоральная модель квазимногосценарной РСЭП;

- разработаны комплексы темпоральных и индикативных лингвистических переменных, отражающих особенности РСЭП; 
- построено нечетко продукционное решающее правило по выбору подсценария, исходя из значений показателей на момент окончания этапа ветвления базового сценария;

- предложено два подхода (на основе полного и неполного нечеткого логического вывода) для построения процедуры выбора подсценариев.
Разработанные формальные описания могут быть использованы при разработке систем поддержки принятия управленческих решений при создании и администрировании выполнением региональных социально-экономических программ.

Исследование выполнено при финансовой поддержке РФФИ в рамках научного проекта № 20-07-00855.

\section{ЛИТЕРАТУРА}

1. Полетаев И.Ю. Организация межведомственного взаимодействия в управлении повышением энергоэффективности региональных программ и проектов / И.Ю. Полетаев // Вестник Российского нового университета. Серия: Человек и общество.— 2020.— № 1.— С. 65-69.

2. Гулиева А.Э.Г. Пути повышения эффективности финансового-кредитного обеспечения региональных программ социально-экономического развития / А.Э.Г. Гулиева // Современная наука: актуальные проблемы теории и практики. Серия: Экономика и право.—2017.—№ 2.—C. 49-53.

3. Lomazov V.A.An assessment of regional socio-economic projects V.A. Lomazov, V.S. Nehotina // Экономика, статистика и информатика.—- Вестник УМ0. — 2013.— № 3.—C. 190-193.

4. Паздникова Н.П. Вопросы оценки социальной эффективности программно-целевого развития региона / Н.П. Паздникова // Вестник Пермского национального исследовательского политехнического университета. Социально-экономические науки. — 2019.— № 1.— С. $219-232$.

5. Полухина М.Г. Экологический менеджмент — основной фактор социально-экономического развития сельских территорий / М.Г. Полухина // Научный журнал НИу итМО. Серия: Экономика и экологический менеджмент. - 2018. — № 2. - С. 91-102.

6. Корниенко В.В. Целевые региональные программы и их роль в социально-экономическом развитии Краснодарского края / В.В. Корниенко, А.В. Сапунов // Modern Science. - 2020.— № 4-3.- С. 107-110.

7. Лякишева В.Г. 06 основных направлениях совершенствования государственной и муниципальной политики в сфере управления транспортным комплексом территории (на материалах Алтайского края)/ В.Г. Лякишева, В.А. Воронкова, В.Р. Сагателян // Экономика. Профессия. Бизнес.-2020.— № 1.- C. 76-83.

8. Ломазов В.А. Применение сценарного подхода при разработке и прогнозировании результатов региональных программ развития агропромышленного комплекса / В.А. Ломазов, О.С. Акупиян, Р.В. Капинос, А.В. Ломазов // Инновации в АПК: проблемы и перспективы. - 2020.— 4(28).- C. $225-238$.

9. Швецова А.А. Эконометрические модели для оценки уровня и темпов развития регионов России / А.А. Швецова // Modern Economy Success. 2020. - № 4. - C. 24-30.

10. Lomazov A.V. Fuzzy formalization of the multi-stage processes temporal description / A.V. Lomazov, V.A. Lomazov, V.V. Rumbesht // Journal of Physics: Conference Series. - 2020.—v. 1661.—Pp. 1-9.

11. Zadeh L.A. The concept of a linguistic variable and its application to approximate reasoning / L.A. Zadeh // Information Sciences. — 1975 — № 1.— Pp. $119-249$

12. Mamdani E.H. Application of Fuzzy Algorithms for Control of a Simple Dynamic Plant / E.H. Mamdani // IEE Proceedings D: Control Theory and Applications. 1974. - 121 (12). - Pp. 1585-1588.

(c) Ломазов Александр Вадимович ( lomazov_a@bsu.edu.ru ). 